\title{
The Distortion Influence Simulation and Experimental Study of Human Body to High Voltage Power Frequency Electric Field
}

\author{
Wang Jingang, Mao Kai and Gao Can \\ State Key Laboratory of Power Transmission Equipment \& System Security and New \\ Technology, Chongqing University, Chongqing 400044, China \\ jingang_023@163.com,173661219@qq.com,343292467@qq.com
}

\begin{abstract}
In order to eliminate the distortion influence error caused by human body on the measurement of hand-held high voltage power frequency electric field measurement device, field intensity distortion caused by induced current of human body has been analyzed, and the enhancement factor of the electric field intensity is introduced to reduce the distortion degree of electric field measurement value by electric field measuring instrument. A simple model of the human body is built, the Ansoft Maxwell is used to simulate and calculate the electric field distribution around the high voltage power frequency transmission lines when human body presented, and distortion influence of field intensity distribution caused by human body has been analyzed. In addition, the enhancement factor value of electric field is determined and the electric field distortion is corrected by experiment and comparing the measurement data with the electromagnetic field analyzer EFA300. With the actual measured values of instrument restored the value of the field intensity when the human body away from the measuring equipment, the distortion influence measurement error can be reduced and measurement precision can be improved.
\end{abstract}

Keywords: Induced current, Ansoft Maxwell simulate, Enhancement factor, Electric field distortion, Electric field restoration

\section{Introduction}

In the power industry, electric field measurement is regarded as effective and practicality means of experimental research [1]. The distribution of electric field around the transmission lines and inside the electric equipment has become an interesting research topic with the continuous improvement of the transmission voltage level. Because of the complexity of human body, dielectric constant is much larger permittivity than that of air. In case of human body being present, the field intensity around the high voltage electric field would be ten times more than that of without human body, and the induced current in the body would be out of the human safety limit [2]. Besides, the surrounding electric field distortion caused by the human body, make the electric field measuring instrument, especially the hand-held instruments can't accurately measure electric field intensity. Therefore, the influences on electric field intensity caused by human body should be considered.

Firstly, according to the theory of electromagnetic fields, effects on measurement of power frequency electric field of high voltage caused by human body under alternating electric field is analyzed. And then, simulation software is used to analyze the distribution of electric field with the human body model which is supposed to be on the ground and directly below the transmission line. Furthermore, field experiment about the actual measurement is carried out, and through the data comparison, correction of field intensity value is done via introduction 
of an enhancement factor. Therefore, effectively reduce the error resulted by the handheld measurement equipment on the electric field with the presence of the human body; eliminate the main factors that cause measurement errors of the handheld measurement device; improve the high voltage transmission line non-contact measurement precision, thus more accurately analyze of power system operation condition.

\section{Theoretical Analysis}

\subsection{Theoretical Condition:}

Assumption the electric field intensity is uniform around the body. For the calculation of human induction current, the induced currents generated by three coordinate components of the space field that act on the human body respectively must be considered and be superimposed to get the total induced current. For each coordinate component of field intensity, it is assumed to be uniform around the human body is uniform [3], and parallel or perpendicular to the axis of the human body [4].

Field intensity around the human body satisfies the quasi static field conditions. Quasi static field refers to alternating electric fields with frequency of $100 \mathrm{kHz}$ and smaller. Dielectric constant of human body is much greater than the air because of its complexity, the human body can be treated as a conductor, the surface electric field intensity vector is perpendicular to the surface of the human body. Consequently, research methods and calculation formulas in electrostatic field can be used here. In addition, charges in the electric field induced by the human body do not affect the charge distribution of transmission lines.

\subsection{Calculation of Current Induced by Human Body}

In power frequency electromagnetic field, the human body can be regarded as a complete conductive body. Generally the body's electrical parameters are:

Permittivity: $\quad \varepsilon=80 \varepsilon_{0}\left(\varepsilon_{0}=8.85 \times 10^{-12} \mathrm{~F} / \mathrm{m}\right)$; resistivity: $\rho=1 \Omega \cdot \mathrm{m}$; permeability: $\mu=\mu_{0}\left(\mu_{0}=12.56 \times 10^{-7} \mathrm{H} / \mathrm{m}\right)$; the relative dielectric constant of the body living tissue is about $1 \times 10^{5} \sim 2 \times 10^{6}$.

Like any conductive object, the whole body is in equipotential state, its surface electric field intensity vector is mutually perpendicular with the human body surface[5], and can be calculated according to electromagnetic field theory [6].

In alternating electric field, the induced charge changes with the electric field, corresponding to the displacement current produced in the human body. In the case of induction electric field intensity given, internal current density can be obtained according to Ohm's law:

$$
\vec{J}=\sigma \vec{E}_{\mathrm{i}}
$$

$\sigma-$ The conductivity of the medium $(\mathrm{S} / \mathrm{m})$

$\overrightarrow{E_{\mathrm{i}}}$ - - Induction electric field intensity $(\mathrm{V} / \mathrm{m})$

Human body total induced current $\vec{I}$ :

$$
\vec{I}=\int_{S} \vec{S} \times \vec{J} d \vec{S}
$$

$\vec{S}-$ - Human skin surface area $\left(\mathrm{m}^{2}\right)$ 


\subsection{Electric Field Intensity Enhancement Factor}

Considering the influence of the human body, usually using the ratio of human body surface electric field intensity $\overrightarrow{E_{\mathrm{s}}}$ to exogenous uniform field intensity $\overrightarrow{E_{0}}$ to describe the relationship between distortion field and undistorted field, known as the electric field intensity increases factor $\partial$ :

$$
\begin{gathered}
\partial=\frac{\overrightarrow{E_{\mathrm{s}}}}{\overrightarrow{E_{0}}} \\
\overrightarrow{E_{\mathrm{s}}} \text { —-The human body surface electric field intensity } \\
\overrightarrow{E_{0}} \text { - }- \text { Outside things uniform electric field intensity. }
\end{gathered}
$$

Theoretical and experimental studies show that the body surface electric field intensity is enhanced in most parts and the strongest locates at the human head.

If the body insulates form the earth, the enhancement factor of human body surface differs. The enhancement factor is about 1 at the foot and lower portion, and about 20 at the head; furthermore, about 50 at the tip of the stretched arm. The results are also shows: the tip of a conductive object would significantly promote the electric field intensity [7].

When the body directly connect to ground, electric field distortion weakens with the same height of the human body surface [8]; but from the human body bottom to the top, enhancement factor increases more than that of insulation form the earth: wherein the enhancement factor about 0.2 at the human foot, and about 17 at the head, we can see the enhancement factor increased 85 times.

\section{Simulation and Analysis on Distorted Electric Field Around the Human Body}

In order to have a better understanding of the influence to the electric field and its distortion with the body under the transmission line, using Ansoft Maxwell software simulates the distribution of the electric field below the transmission line with human body directly connecting to the earth.

\subsection{Assumption of Parameters Related to Human Body:}

Because the ground electric field can actually be considered uniform below transmission lines, and perpendicular to the ground [9]; and to ignore human induction current bring electromagnetic interference, etc. [10]. Therefore, a pair of parallel electrode plate applied with voltage can be regarded as the equivalent electric field of transmission line.

Suppose the distance between the two plates is $2 \mathrm{~m}$, and the plate voltage of high voltage is $8 \mathrm{kV}$, low voltage plate and the earth potential is set to $0 \mathrm{~V}$, ignore the edge effect, the electric field between the two plates can be considered to be uniform, which is $4 \mathrm{kV} / \mathrm{m}$. For convenience, treat the human body as a conductor model and divide the human body model into four parts, the head, neck, waist and legs [11]. Wherein the head is equivalent to a radius of $9 \mathrm{~cm}$ sphere, the neck, waist and legs are equivalent to a cylinder, the specific parameters are as follows: neck radius is $6 \mathrm{~cm}$, and the waist radius is $20 \mathrm{~cm}$, leg radius is $15 \mathrm{~cm}$; neck height is $8 \mathrm{~cm}$, and waist height is $60 \mathrm{~cm}$, the leg height is $90 \mathrm{~cm}$. The total height of the human body model is $176 \mathrm{~cm}$. Suppose the conductivity of the human body is $0.1 \mathrm{~S} / \mathrm{m}$, the relative 
dielectric constant is about $10^{5}$, human body and the earth are equal potential [12]. Simplified human model structure is shown in Figure 1.

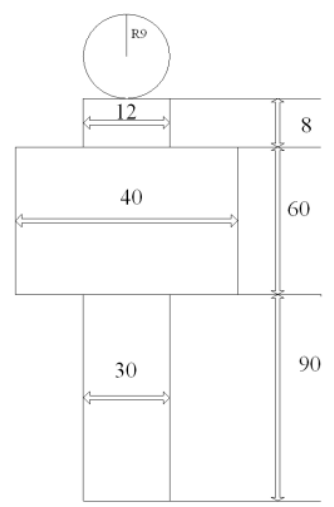

Figure 1. Structure of simplified human model

\subsection{Simulation Analysis}

The equivalent electric field considering the presence of human body below the transmission line can be obtained via simulation using Ansoft Maxwell.

Analysis shows: the existence of human body disturbs the electric field in a certain range. The overall trend of electric field distortion is that the distortion effect and the enhancement factor increase with the height of human body. From the soles of the feet to the top of the head, the electric field intensity ranges from $7.91 \mathrm{~V} / \mathrm{m}$ to $79.1 \mathrm{kV} / \mathrm{m}$. Wherein the maximum field intensity at the top of the head is about $79 \mathrm{kV} / \mathrm{m}$, enhancement factor is 19.75 with respect to undistorted maximum field intensity that is $4 \mathrm{kV} / \mathrm{m}$ : waist middle position field intensity is about $11 \mathrm{kV} / \mathrm{m}$, the enhancement factor is about 2.75 ; the field intensity of ground position place is $7.91 \mathrm{~V} / \mathrm{m}$, close to 0 , but the field intensity remain has been increased relative to undistorted situation.

As can be seen from the simulation analysis, the head of human body has the largest electric field intensity. This is because the human body can be approximated as a grounded conductor for a very large, the top of the head are closest to the positive plate, and gathers a large number of induced charge, so there is a maximum field intensity. With the height reducing, the further the body from the positive plate, the less induced charge, so, the field intensity decreases. Furthermore, due to the effect of electrostatic induction, charges distributed in the outermost surface of the human body. So the human body such as the inner concave place of the neck, the electric field is very small, and such as the outer convex place of shoulder, the electric field is large. Although simplified human body model is difficult to fully simulate many concave and convex parts of human body, the overall distribution of the electric field trend will not be affected by these parts. Therefore, enhancement factor generally decreases with the decreasing height of human body.

\section{Field Testing}

\subsection{Electric Field Intensity Test Equipment}

Use the EFA300 low frequency electromagnetic field analyzer in the testing made in German Narda Company. EFA300 equipped with three-dimensional isotropic measurement probe (non-directional), electric field measuring range is $0.1 \mathrm{~V} / \mathrm{m}-200 \mathrm{kV} / \mathrm{m}$ [13]. Electric field measurement value is more accurate in the low frequency high voltage [14]. 


\section{2. $500 \mathrm{kV}$ Ban Chen II Line Field Testing}

In order to more accurately analyze the characteristics of the distortion of electric field around the human body, we had carried out experiments on the distorted electric field around the human body in Chongqing University Town $500 \mathrm{kV}$ Ban Chen II line. Measured body is insulated from the ground, measures the surface electric field of the five main parts, the human head, middle of the neck, middle of the waist, middle of the legs and foots, respectively. The whole measuring process considering undistorted electric field to be unchanged and uniform. Field measurement is shown in Figure 2.

And selected one measuring point at the transmission lines roadside phase outer, the actual measurement value of undistorted electric field is $4.048 \mathrm{kV} / \mathrm{m}$ where the point is $1.5 \mathrm{~m}$ above from the ground.

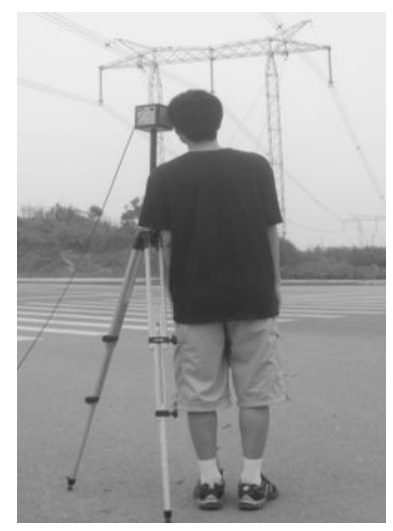

\section{Figure 2. Scene of field measurement}

\subsection{Test Analysis}

Through finishing the measurement data, the relationship curve between the human body height and the electric field enhancement factor in case of body insulating from the grounded is shown in Figure 3.

It can be seen from the relationship curve, when the human body is below the transmission lines, the higher the height, the closer the head to the line, so both the electric field of the head and the enhancement factor are bigger; on the contrary, because the shorter person a little far away from the line, the enhancement factor is smaller. Similarly, other parts of the body's electric field and enhancement factor can be analyzed.

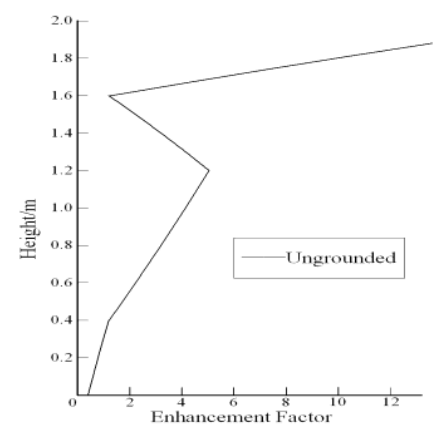

Figure 3. Enhancement factor curve of ungrounded human mode 
In addition, the body height and the electric field enhancement factor relationship curve in Figure 3 are basically consistent with the test results in literature [2], but the enhancement factor size still have slight difference. This is because the close relationship between the height and the distortion electric field caused by human body [15]. The higher the measured human body, the smaller distance from its head to a charged object, then the greater the field intensity; at the same time, different people different body model, and dielectric constant and conductivity will also differs, so the distortion of electric field will also be different; Furthermore, due to the distortion electric field caused by the body also associated with the body's physical fitness, body size, and other factors [16], it will bring some error in the analysis process.

\subsection{Calibrate Reduction Test to Electric Field}

Considering influence on the body's distortion influence situation, introduce corresponding increase factors to conduct calibration test to of electric field distortion. We also carried the field measurement in Chongqing University Town. Choose a measuring point carry out two groups of field intensity data measurements at the transmission line roadside phase outer, wherein a group of people tight close to the measuring instrument. At the same time it should be noted that, don't change the instrument measuring height and position in the measurement to ensure the accuracy of reading. Two groups of instrument field measurement results and simulation results contrast as shown in Figure 4. From the Figure 4, two groups of measurement results are closely similar, measurement error is mostly within $10 \%$ and meet the requirements of the engineering measurement.

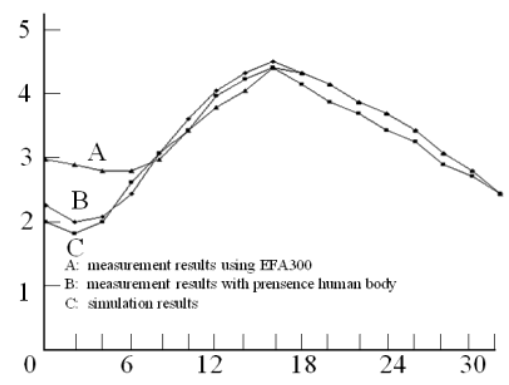

\section{Figure 4. The Comparison of the vertical electric field between measurement} and simulation below 500kV transmission line

\section{Summary}

Based on the theory analysis and the actual measurement indicate that the human body surface electric field intensity is enhanced in most parts. The enhancement factor is not same in different positions, which minimum is in a human foot or low parts and maximum is in the head. If the tip of stretched out arm is over the head, the enhancement factor will increase. In general, enhancement factor reduces with the reducing height of human body.

In order to make the measured value close to undistorted the real data to the utmost, through several comparison measurements, introduce the corresponding enhancement factor to calibrate electric field distortion, to make the measurement value fully reflect the undistorted field when someone presented, effectively reduces the influence of the electric field measurement result due to the human body, and improve the accuracy of the electric field measuring instrument, especially the accuracy of the electric field measurement of the hand-held electric field measuring instruments. Improve the non-contact type measurement 
accuracy of high voltage transmission lines, thus more accurate analyze the operation status of power system.

\section{Acknowledgements}

This work was supported by Natural Science Foundation Project of China (Grant No. 51207175).

\section{References}

[1] L. Conghan, H. Wei and W. Jingang, "Design of portable power frequency electric field measurement device" High Voltage Apparatus, vol. 48, no. 3, (2012), pp. 57-61.

[2] L. Yuanqing, Z. Jun and Z. Bo, "Analysis of influence of $50 \mathrm{hz}$ electric fields generated by the high voltage power lines on human body", High Voltage Engineering, vol. 30, no. 12, (2004), pp. 35-36, 55.

[3] J. Wei, W. Guangning, H. Zhen, "Analysis of effects on human body of UHVDC transmission line based on FEM" High Voltage Engineering, vol. 9, (2008).

[4] F. Yanyan and Y. Jihui, "Power frequency electric field cause by EHV lines and its effect", Journal of Chongqing University, vol. 27, no. 3, (2004), pp. 10-14.

[5] A. Z. El Dein, M. A. A. Wahab and M. M. Hamada, "Computation of electric field and human body induced current under overhead transmission lines", 2010 7th International Multi-Conference on Systems, Signals and Devices, (2010), pp. 1-6.

[6] C. Dingxin and Q. Xuedi, "Evaluated calculation of the induced current in human body produced by power frequency electric and magnetic field under ac transmission line", Guangdong Power Transmission Technology, vol. 4, (2010).

[7] A. Chiba, K. Isaka and Y. Yokoi, "Application of finite element method to analysis of induced current densities inside human model exposed to $60 \mathrm{~Hz}$ electric field", IEEE Trans on PAS, vol. 103, no. 7 , (1984), pp. 1895-1902.

[8] M. Abdel-Salam and H. M. Abdallah, "Transmission-line electric field induction in humans using charge simulation method", IEEE Transactions on Biomedical Engineering, vol. 42, no. 11, (1995), pp. 1105-1109.

[9] H. Jiusheng, "Possible effects of electromagnetic fields generated by electrostatic discharge from the charged human body on computer operation", Physics, vol. 10, (2000).

[10] L. Qin, "Electromagnetic interference effects and restraint strategy on electronic electric energy meters", Electrical Measurement \& Instrumentation, vol. 44, no. 6, (2007), pp. 50-54.

[11] Z. Songyang, Y. Degui and L. Qing, "Development of wireless power frequency electric field measurement and alarm device and its application", Electrical Measurement \& Instrumentation, vol. 48, no. 1 , (2011), pp. 21-25.

[12] Z. Tiebin and M. C. Comber, "Calculation of electric field and potential distribution along nonceramic insulators considering the effects of conductors and transmission towers", IEEE Transaction on Power Delivery, vol. 15, no. 1, (2000), pp. 313-318.

[13] G. H. Varttaneourt, S. Carignan and C. Jean, "Experience with the detection of faulty composite insulators on high-voltage power lines by the electric field measurement method", IEEE Transactions on Power Delivery, vol. 13, no. 2, (1998), pp. 661-666.

[14] S. Fangyin, B. Kehan and Z. Liurong, "Electric field distribution and human induced current on the $220 \mathrm{kV}$ to $500 \mathrm{kV}$ transmission line tower", Power System Technology, no. 1, (1992), pp. 53-59.

[15] M. Misakian and P. Fulcomer, "Measurement of non-uniform power frequency electric fields", IEEE Transactions on Electrical Insulation, vol. EI-18, no. 6, (1983), pp. 657-661.

[16] E. Lanbert, "Measurement of electric and magnetic fields from alternating current power lines", IEEE Transactions on Power Apparatus and Systems, vol. PAS-97, no. 3, (1978), pp. 1104-1114. 
International Journal of Control and Automation Vol.6, No.6 (2013) 